\title{
GoSoapBox in public health tertiary education: A student response system for improving learning experiences and outcomes
}

\author{
Julie-Anne Carroll \\ Queensland University of Technology \\ Mangalam Sankupellay \\ James Cook University \\ Michelle Newcomb, Jess Rodgers, Roger Cook \\ Queensland University of Technology
}

\begin{abstract}
Most pedagogical literature has generated "how to" approaches regarding the use of student response systems (SRS). There are currently no systematic reviews on the effectiveness of SRS, for its capacity to enhance critical thinking, and achieve sustained learning outcomes. This paper addresses this current gap in knowledge. Our teaching team introduced GoSoapBox (an interactive online SRS) in an undergraduate sociology and public health subject, as a mechanism for discussing controversial topics, such as sexuality, gender, economics, religion, and politics, to allow students to interact with each other and to generate discussions and debates during lectures. Bandura’s Social Learning Theory (SLT) was applied to investigate the effectiveness of GoSoapBox for improving learning experiences. We produced a theoretical model via an iterative analytical process between SLT and our data. This model has implications for all academics considering the use of SRS to improve the learning experiences of their students.
\end{abstract}

\section{Introduction}

There is currently a wide range of research into the recent introduction of student response systems (SRS) in tertiary settings (Banks 2006; Beatty \& Gerace 2009; Kay \& Le Sange, 2009; Lantz, 2010; Sprague \& Dahl, 2009). However, most of this pedagogical literature has generated how to approaches regarding the use of clickers, and similar response technologies. There are currently no systematic reviews on the effectiveness of GoSoapBox - a newer web-based, popular SRS. With rapid developments in teaching and learning technologies across all undergraduate disciplines, there is a need to obtain comprehensive, evidence-based advice on these types of technologies, their uses, and overall efficacy. This paper addresses this current gap in knowledge.

Our teaching team from the Queensland University of Technology, introduced GoSoapBox in an undergraduate 350 student cohort, public health subject. First, this paper will cover the recent literature reviewing student response systems in tertiary settings. Second, we describe the methods used to implement the use of GoSoapBox in our lectures and tutorials, and which aspects of the technology we drew on for learning purposes, as well as the methods for obtaining feedback from the students about the effectiveness of this tool. Third, we will outline the theoretical framework used to generate this pedagogical research. In keeping with the social and collaborative features of Web 2.0 technologies, Bandura's Social Learning Theory (SLT) will be applied here to investigate the effectiveness of GoSoapBox as an online tool for improving learning experiences and the quality of academic output by students. Bandura has emphasised the Internet as a tool for self-controlled learning (Bandura, 2001), as it provides the education sector with an opportunity to reconceptualise the relationship between learning and thinking (Glassman \& Kang, 2011). Next, we report findings and analysis that are produced as a result of an examination of all student and staff activity on GoSoapBox as well as reports from students about the benefits and limitations of it as a learning aid. Finally, we display a theoretical model that is produced via an iterative analytical process between SLT and our data analysis for use by academics across the undergraduate curriculum. 


\section{What are SRSs?}

SRSs are systems consisting of wireless handheld devices and presentation software that allow students to answer questions in real time (Méndez-Coca \& Slisko, 2013). They might be called clickers, key-pads, handsets, or zappers (Caldwell, 2007; d’Inverno, Davis, \& White, 2003). In tertiary settings, these devices have been traditionally supplied by universities for student use within classes. However, they are limited to yes/no or multiple-choice polls, unlike recent technological advancements which have led to the adaptation of web-based SRSs, such as Socrative (www.socrative.com), Piazza (https://piazza.com), Poll Everywhere (www.polleverywhere.com), Hotseat (https://www.learntechlib.org/p/106969), and GoSoapBox (http://www.gosoapbox.com/).

The newer web-based SRS allow for large cohorts and classrooms of tertiary students to engage in real time online questions and debates without the need for inbuilt hardware, using their own mobile devices as mechanisms for engagement. Unlike traditional SRSs, web-based SRSs accommodate both multiple choice and opened question (Méndez-Coca \& Slisko, 2013). The use of such systems enables educators to create a learning environment based on content questions but can also seek their views on wider, social and contextual issues. Web-based SRSs instinctively appeal to the current cohort of undergraduate students, below the age of 25 (Hoppenfield, 2012). This generation, the digital natives, are constantly immersed in technology, accessing information in the format of text, audio, or video, playing games, or communicating via social networking web sites on their computers or mobile phones (Hoppenfeld, 2012). Matthew and Evans (2012) found that $86 \%$ of their students use social media. This enthusiasm for social media when combined into learning experiences, such as SRSs, results in improved frequency and quality of interactions encouraging a collaborative active learning environment (Junco, Heibergert, \& Loken, 2011).

\section{What is GoSoapBox?}

GoSoapBox is a web-based SRS platform available via license to educational institutions. Lecturers or tutors signup for GoSoapBox accounts to set up sessions given a unique log-in code and students then access each session via the log-in code. GoSoapBox functions include multiple-choice polls, open-ended discussion questions (set up by the instructor), and social chat, where students could pose questions or start discussions on anything they wished. Participants can vote up these open discussions, perhaps demonstrating that it is a good discussion topic, question, or they agree with the statement. All contributions are anonymous to other students, but staff can select if they can view the names of contributors.

\section{GoSoapBox in Public Health Unit: PUB209, Health, Culture, and Society}

Health, Culture, and Society PUB209 is a 350 student first year core unit for a broad range of health disciplines, including paramedic, psychology, nutrition and dietetics, nursing, and public health. The unit focuses on the study of the social and cultural dimensions of the human body, mind, and health. We introduce public health from sociological and anthropological perspectives, with a core emphasis on the ways in which social, cultural, political, and economic systems shape human health behaviours and outcomes. The unit introduces students to the ideas and writing of key social theorists as they relate to health systems, behaviours, and outcomes. We examine the practical relevance of these theories in relation to understanding complex phenomena, such as cultural safety, risk-taking behaviours, happiness, health, life-expectancies, and death. We examine direct and indirect links between ethnicity, class, gender, sexuality, geography, and health, with the following research questions:

1. What is the extent and level of engagement of students with a SRS such as GoSoapBox during the delivery of lecture content to a large, multidisciplinary undergraduate cohort?

2. In what ways do the qualitative components of GoSoapBox expose the learning engagement processes of students in a socially and culturally sensitive public health unit?

3. Can we apply a SLT to unearth constructivist processes generated by GoSoapBox in terms of reciprocity and modelling to aid discussion, engagement, and understanding amongst students? 


\section{Methodology}

We conducted a survey to gauge the extent of the usage by students of the GoSoapBox. This initial data collection produced a set of descriptive statistics regarding the uptake and use of this new learning technology by our cohort. We then analysed the qualitative data produced by the students in GoSoapBox during and between weekly lectures. We applied Bandura's SLT on iterative psycho-social processes to the analysis of this data. We applied this framework due to its compatibility with the technological, social, and pedagogical nature of GoSoapBox, in that it is interactive, and open to reading and observation by all students. The theoretical framework as it applied to the data collection and analysis is outlined below.

\section{Theoretical framework}

In keeping with the social and collaborative features of Web 2.0 technology, Bandura's SLT (1977) was applied here to investigate the effectiveness of GoSoapBox as an online tool or mechanism for improving learning experiences and in-class student engagement. The premise of SLT is that an individual's cognition, behaviour, and social environment continually interact, in a reciprocal, iterative, and cyclic fashion (Bandura, 1977). Bandura has emphasised the Internet as a tool for self-controlled learning (Bandura, 2001), as it provides students with an opportunity to reconceptualise the relationship between learning and thinking (Glassman \& Kang, 2011). A substantial body of pedagogical research investigating social processes in learning contexts have successfully used SLT to enhance an understanding of reciprocal and modelling relationships in building knowledge (Chiu, 2014; Corriveau, 2016; Hauffman et al., 2017). Specifically, Corriveau (2016) used the basis of SLT to find out whether an active or passive informant was preferred amongst young children when deciding who to learn from when give instructions. Hauffman et al. (2017) generated and evaluated an internet-based intervention between patients with cancer, clinicians, and researchers to enhance information gathering and self-care based on SLT. They found the interactive and modelling components described by Bandura, and which are available through Internet-based interaction to be particularly useful in creating a successful program. Further to this, Chiu (2014) explored Bandura's widely referenced concept of self-efficacy (Alqurashi, 2016; Bandura, 2012; Snyder \& Fisk, 2016), which is an inherent component of the motor reproduction phase in SLT, to further understand relationships between stress and Internet addiction in university students.

Bandura argues that human behaviour is not inbuilt as such but, is learnt, both via one's own experiences and via the interactions and modelling of others in the human social world. Through the process of modelling other people's behaviour, individuals begin to cognitively grasp strategies for carrying out a new, or learnt, behaviour. By observing and interacting with others, Bandura argues, humans develop knowledge, which they use to inform their future actions and behaviours. Bandura describes this complex, interactive process of social learning as being comprised of four key conceptual elements: (1) attentional processes, (2) retentional processes, (3) motor reproduction processes, and (4) motivational processes. This theoretical framework, and specifically these learning processes described by Bandura, provided the lens for examining and analysing the types of interactions that occurred during in-class GoSoapBox. The four components of the theory are defined below:

1. Attentional processes: In order to observe accurately, attention needs to first be given to the behaviour. If the behaviour is seen as attractive, the individual is more likely to give it attention. An individual may be more inclined to pay attention to a modelled behaviour that they will be required to perform publically, rather than privately.

2. Retentional processes: An accurate reproduction of the modelled behaviour may be more likely if the behaviour is immediately imitated, if the observer is repeatedly exposed to the particularly behaviour, and/or if the actions required to complete the behaviour are rehearsed, mentally and then overtly.

3. Motor reproduction processes: To convert knowledge into behaviour, the required actions need to be cognitively selected and organised, spatially and temporally. The course of action that an individual chooses to take is largely dependent on the anticipated outcome and the confidence that they possess in their own ability to perform the necessary actions (self-efficacy). Self- 
efficacy will dictate, at least in part, the level of difficulty they wish to tackle, and the amount of effort they wish to invest.

4. Motivational processes: People are also more likely to model behaviours that lead to pleasing results (Bandura, 1977). Differential reinforcement by models, is likely to lead to appropriate behaviour. If a model gives similar feedback to everyone performing the behaviour, regardless of the quality, the behaviour is not imitated well (Bandura, 1977). The provision of models in learning may allow "novices" to accurately carry out a desired behaviour. Indeed, those who lack confidence, may benefit the most from models. The provision of a range of models, may lead to creativity and innovation.

\section{Data collection and analysis}

\section{Ethics}

Formal research ethics approval was not required (personal communication with university ethics committee), as the primary purpose of this study is teaching and learning quality assurance, and as a result does not require university human research ethics review, approval, and monitoring. The study was, however, conducted in strict accordance with the National Statement, university policies and legislation. Participation in within the in-class GoSoapBox activity was voluntary and occurred during semester. Students were advised that their comments were not linked to their student or personal identity in any way and were anonymous and public. All survey and quantitative data was also anonymous and not linked to student nor personal identity.

\section{Phase 1: Quantitative data}

A GoSoapBox session was set up to survey students on their opinions on the use of GoSoapBox throughout the semester and how they saw it as a platform that contributed to learning. This generated a meta-use of GoSoapBox to monitor the students reflective processes behind their active contributions to class discussion on the weekly content-based GoSoapBox sessions, which were activated during live lectures.

\section{Phase 2: Qualitative data}

In reference to Bandura's SLT as a mechanism for analysing our data, a social constructionist methodological approach suited our focus. Social constructionism is a theoretical framework that specifically acknowledges the human communication and interaction that goes on over time to construct agreed upon norms and appropriate or acceptable behaviour in any given context (Berger \& Luckman, 1966). We considered social constructivist elements of the student/teacher learning processes as well, which draws a focus to the iterative and cyclical nature of communication between peers and teachers that goes on over time to ensure a dynamic of multi-directional, reciprocal learning contexts (Schunk, 2012). We conducted a thematic analysis in the first instance, followed by axial coding to explore the relationships between the key concepts arising from the data. Axial coding was undertaken by examining the theoretical relevance of the arising themes to the research questions, and to explore the relationships between them. Axial coding shifted the key categories from themes into theoretical building blocks that we related back to our SLT framework, and with which we worked to develop our model to explain how the collaboration, discussion, and the learning worked amongst students. We employed SLT to organise the emanating findings from a social constructionist perspective, and to develop theory about how ICTs, such as GoSoapBox, work to increase engagement, discussion, debate, and to improve in-class experiences. We paid particular attention to the social dynamics that shaped the students' internal processing and outward performance during these interactions and debates. This approach helps to balance the methods and findings that can be triangulated across the different data sets.

Figures 1 and 2 are examples of GoSoapBox Polls, Discussion, and Social Q\&A sections posted by teaching staff and responses by students throughout the semester. 


\begin{tabular}{|c|c|c|}
\hline \multicolumn{2}{|c|}{20 GoSoapBox } & S Jey \\
\hline \multicolumn{3}{|c|}{ PUB209 Lecture Five } \\
\hline \multicolumn{3}{|c|}{ Polls } \\
\hline \multicolumn{3}{|c|}{ A focus on profit and efficiency... } \\
\hline \multicolumn{3}{|c|}{ I feel that the university experience... } \\
\hline \multicolumn{3}{|c|}{ I think that getting rid of bulk billing is.... } \\
\hline \multicolumn{3}{|c|}{ Should everyone be covered for the cost of ambulance trips? } \\
\hline \multicolumn{3}{|c|}{ Discussions } \\
\hline \multicolumn{3}{|c|}{ Do you consider people's working conditions when you purchase clothes/goods? } \\
\hline \multicolumn{3}{|c|}{ One day all degrees/courses will be delivered online and this is an example of McDonalisation. } \\
\hline \multicolumn{3}{|c|}{ University should be free and entry based on merit, not means. } \\
\hline \multicolumn{3}{|c|}{ What are some other examples where you see the McDonalisation of society going on? } \\
\hline \multicolumn{2}{|c|}{ Social Q8A } & † Ask \\
\hline 슨 & What is the difference between "Globalisation" \& "McDonalisation" ? & \\
\hline
\end{tabular}

Figure 1. Example of GoSoapBox Polls, Discussions, and Social Q \& A posted by teaching staff

What are some other examples where you see the McDonalisation of society going on?

Give examples, and your opinion on the ethical considerations we should be making about these examples, if any.

\begin{tabular}{l} 
Replies \\
Internet dating \\
:-) Love this comment. J-A \\
Hungry Jacks \\
self serve checkouts \\
Aged Care \\
Aged care. Another good one. J-A \\
GP super clinics \\
Beauty. More cookie cutter than sausage factory, but the point could be argued... \\
airports! \\
mining - arent the truck drivers going to get replaced with automated mining trucks so that the risk \\
associated with mining jobs is eliminated and those who worked as drivers will be trained in other \\
sectors but with a much smaller pay rate. \\
\hline
\end{tabular}

Figure 2. Examples of responses written by students on GoSoapBox 


\section{Results}

\section{Quantitative findings}

Around 50\% of the students surveyed stated that GoSoapBox positively influenced their choice to attend lectures, while the other half claimed they were not influenced. None of the students were put off from attending the lectures due to GoSoapBox usage. Approximately a third of the students (32\%) stated that the best thing about GoSoapBox was keeping them engaged in lectures, a third of the students enjoyed the social and interaction aspects during lectures, and a third of the students utilised GoSoapBox for learning new concepts and developing their critical thinking skills. The majority of the students (51\%) found the Discussions component of GoSoapBox was the best component to their learning, followed by Polls and Social Q\&A. About 10\% of the responses stated that GoSoapBox did not help at in their learning process. Around $60 \%$ of the students found GoSoapBox to generate an "unsafe space" or offensive environment sometimes, but felt it was good to hear different opinions. This didn't put them off from attending lectures and participating in the Discussions and Social Q\&A component of GoSoapBox.

\section{Qualitative findings}

The SLT framework has found that it is crucial for a diverse range of models and for a wide range of responses to be present if students are to gain both the confidence and the ability to participate in discussions that lead to sustained learning and improved critical thinking. A conceptualisation of how the four elements were able to draw out the learning processes inherent within the context of GoSoapBox in lectures is outlined below and summarised in Figure 3.

\section{Attentional processes: Selecting desirable communication and interactions}

Bandura's first stage of the SLT model, attentional processes, involves the social and psychological processes of selecting which behaviours one pays attention to in order to learn from them, imitate them, and re-model these behaviours appropriately. He claimed that people are more likely to pay attention to behaviours that are seen as desirable and ones that the individual knows they will have to perform at some stage themselves within a public space or social arena. The attentional processes undertaken via students during the GoSoapBox sessions were elicited both when they were requested to make a contribution to polls and discussions, and again when reflected in the comments students made when they gave feedback about the usefulness of this as a learning and interactive tool.

In relation to answering the question about the extent of student engagement with GoSoapBox, it became apparent that students were paying close attention to what was being written during class, as the class would react simultaneously through laughter or whispering or louder in-class chatter as a diverse range of comments and thoughts were shared in this online public space. We were able to gain insight into which comments were receiving the most attention via the voting up system available on GoSoapBox. For example, comments that were seen as desirable in their capacity to invoke critical thought, debate, thought-provoking philosophies, or were humorous, generally received the most attention, and were given the most votes by the students. For example, the questions: "How do I come out of the closet?", "Why do we hate spirituality so much in Australia?" and "Do you think people will laugh at what we believe in 600 years from now?" were popularly received and students gave these the most attention via this online voting system.

Aside from this quick-response voting feedback system, students expressed that they preferred some comments over others. They also explained why they valued some of the comments, and what they gained out of having access to the thought processes and intellectual and social responses of their peers to the traditional lecture format. One student stated: "It was great getting to read a point of view I hadn't thought of." Another noted: "Some of the funny things people wrote made boring parts of the lectures more bearable." Students were discerning in terms of what they chose not to pay attention to, where for example: "[Some students made] immature and offensive comments." Another student stated that they were annoyed by "people asking irrelevant questions, looking to get a laugh by submitting something offensive and/or stupid." While another expressed this view about negative or unhelpful comments during lectures: "Having to read offensive comments by ignorant individuals - you think you're funny - you're not." 
These feedback responses gave the teaching staff insight into how some behaviours were selected and viewed as desirable and worthy of attention during class, and some were shunned or ignored by students. Because this process occurred in a public, online forum, this feedback was provided immediately and directly to those generating the discussions, thus shaping the nature and content of interactions relatively quickly. Students were able to directly control acceptable norms for interaction and discussion during class by selecting which behaviours were given attention, and which were filtered out. This suggests the large capacity GoSoapBox holds to encourage iterative, social constructivist learning processes during live lectures, that lecturers with opportunities for learning about the critical thinking within their cohort, and students the chance to develop norms for contexts of social learning experiences. Interestingly, some students had the confidence to defend their contributions, even when they were deemed less popular, or received less attention, for example: "People getting their heads ripped off for their opinions. They may or may not know better than you, they're not all trolls.”

\section{Retentional processes: Observing, learning, and modelling interactive learning}

The retentional process, as described in SLT, is the provision of circumstance or context for learning wherein behaviours are able to be viewed regularly and repeatedly, and can be rehearsed mentally prior to any attempt at modelling or performance. This process was maximised in three ways via the GoSoapBox interactive tool. Firstly, students were able to observe for as long as they liked before deciding whether or not to participate in the discussions, staying in the attentional phase for as long as they needed before attempting to join in and contribute to the online debates and comments.

Secondly, the fact that GoSoapBox was practiced within a written, rather than a spoken medium, allowed students the opportunity to edit their thoughts prior to exposing them to their peers. Contrary to in class discussions, where students make verbal contributions that are very difficult to alter in the moment, or to retract, these comments could be reviewed, edited, or deleted by the person producing them - thus allowing more of a rehearsal time during the practicing of contributions to debate and discussion between academics and peers. It also allowed comments to be made anonymously, and people could express their ideas without revealing their identity, as one student explained: "It was good getting to make jokes behind a mask.”

Thirdly, students gained an additional opportunity to observe and analyse communication and debate between their peers and to see a sharing of ideas that they would otherwise not have any access to. One student state: “It opened my eyes up to differing opinions. I wasn’t so much offended by things I strongly disagree with, but was reminded by how much viewpoints can vary by." Another student stated that GoSoapBox "opened up good discussions ... and gave feedback about what others were thinking. Getting other's perspectives on a chosen topic. I found their comments and feedback really interesting."

This may have contributed to their confidence in the tutorials that followed, as GoSoapBox appeared to offer a form of online demonstration of how these debates could occur, the direction their peers prefer them to take, the valuing of some comments over others, and most importantly to gain insight into how their peers were experiencing the lecture, and their responses to what they were hearing. The qualitative components of GoSoapBox investigated via our second research question, found that students felt that it was able to make a good connection between the lecture and tutorial contexts, aiding and stimulating discussions in the process: "My tutor used it but we used it as a group to analyse the general consensus in polls. It helped initiate further discussions on the topic.” These opportunities appeared to greatly influence the perceived quality and enjoyment involved in the learning processes, and these students expressed: "In lectures it was amazing, it made my experience just a thousand times greater" and "GoSoapBox was great in lectures because you got to communicate with the whole group."

\section{Motor reproduction}

The process of motor reproduction within the context of learning environments involves a range of decisions students make about whether or not to participate or to attempt to undertake the cognitions they have been processing mentally. These decisions are usually based on whether an individual perceives it to be worth their time and effort, and whether or not they have the competence and skills-base to perform socially or publicly. This applies particularly pertinently to the GoSoapBox environment, because 
although the comments made by students are anonymous, they are viewed and consumed with the entire class. They are also rated by others in terms of voting up or simply responding to the thoughts and ideas that have been put forward. The types of comments and questions students contributed ranged from content-related critical thinking to superficial humour, and in some cases offensive and antagonistic responses, often termed trolling.

In further addressing the qualitative processes in GoSoapBox, there appeared to be a core group of individuals who would participate most frequently, and were the least inhibited in asking controversial or challenging questions, or even to make comments or ask questions that they knew would be received in a negative light. For example, some of these types of comments and questions included:

Yeh [Australia] still is sexist, but the moment you allow yourself to become a victim, the moment you give power to the people. Instead of complaining about things, get up and work for a change and stop playing victim - Burn your bra, prove your point as an individual.

Regarding Australia’s asylum seeker policy, one student stated: "Sink boats until they realise resistance is futile!!!” Regarding legal ages of consent: "If there's grass on the field, play ball” and "Old enough to bleed, old enough to breed."

What GoSoapBox seemed to offer the cohort of undergraduate students, was an opportunity to contribute to socially to lecture content that is usually consumed silently by each individual student, and to ask questions and share ideas that would have otherwise only been processed internally. This sharing of ideas in this context was well received, as this student explained: "I found it really interesting to witness an uncensored anonymous conversation between my peers.”

Those confident enough to perform and contribute to the GoSoapBox debates were well-received by the other students, even if they did not always agree with their thinking, as this comment demonstrates: "[GoSoapBox is a] great way to see every perspective and think about things at a deeper level" and "You would never normally get to here [sic] such strong opinions from these people.”

\section{Motivational process}

Our findings indicate that the presence of such a diverse range of comments and questions on GoSoapBox - which we attribute to anonymity and immediacy of the delivery - students were more likely to be exposed to models and examples that they would not ordinarily experience in the traditional, formal classroom format. In addition to gaining access to what are usually processed as individual, internal thoughts of other students, the teaching staff present in the lecture audience also participated in online discussions. Students engaged with debate with each other, but this was often moderated and mediated by teaching staff who were either in the classroom at the time or in remote locations, and who had logged into the online session.

In answering our third research question regarding the applicability of SLT to this particularly learning context, we found that GoSoapBox gave students an opportunity to ask academic questions as the lecture was progressing, and to challenge what was being said in a relatively safe context. Students asked questions, such as: "Do left and right wingers see eye to eye on many issues or is it simply black and white with their values and decisions on policies?" and "What's the difference between Globalisation and McDonaldlisation?" They challenged what they felt was a theme throughout the unit: "I think we should have had speakers both from left and right wing for a more complete view of issues" and "There is a moderate left vibe coming from this class I must say.”

They were moderated in their behaviour if it was seen to be inappropriate or offensive. On occasions more than one staff member would join in to moderate a debate or mediate an argument, for example:

To the person who complained about the Transgender lecture - it's the 21st century, we have transgender, lesbians, gays, cisgender and others. Deal with it. (Student)

So you're saying you're intolerant to intolerance? (Teacher) 
Intolerance isn't a free pass to say what you want and not have people respond or call your thinking outdated. (Student)

I found a lot of the information unnecessary and confronting especially information about the lecturers personal views and personal life. We shouldn't have to listen to that. (Student)

If you found it confronting it might be useful to think about why, and integrate that into your learning reflections. She didn't say anything offensive - if you were offended maybe consider why. She is not heterosexual and her son is transgender. I am not sure how this is confronting? However it is important to reflect on your reactions. It's all important in terms of learning. (Teacher)

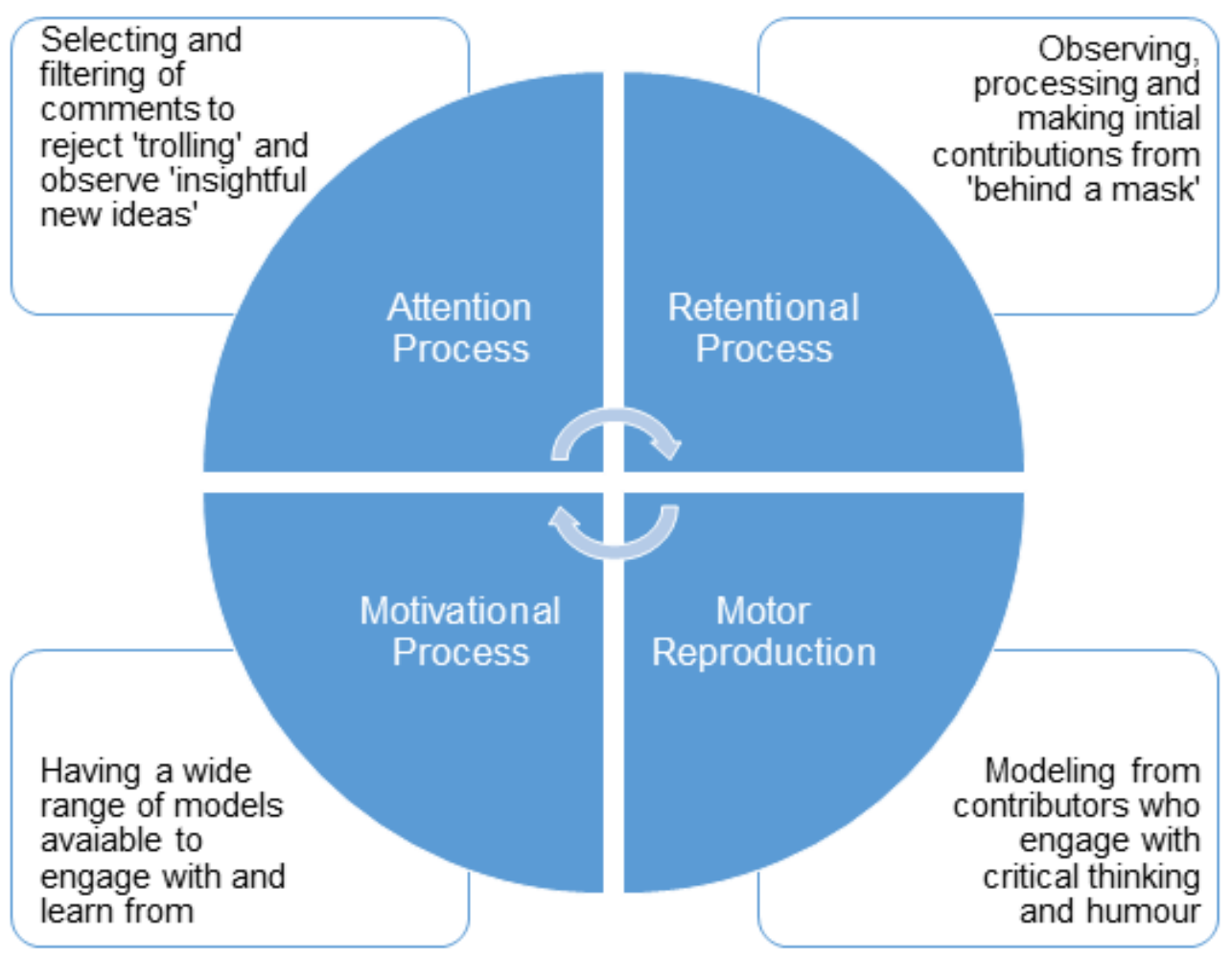

Figure 3. Interactive process of social learning comprises of four key conceptual elements: (1) attentional processes, (2) retentional processes, (3) motor reproduction processes, (4) motivational processes.

\section{Limitations of GoSoapBox as a learning engagement tool}

Anonymity can encourage certain kinds of behaviour; it can inspire confidence to comment in those who wouldn't normally share their opinions out of fear of official or social repercussions (Reader, 2012). Anonymity can encourage broader participation in discussion of topics which are sensitive (Reader, 2012), a particularly important point for our analysis. GoSoapBox topics included discussions on race, class, ethnicity, gender, and sexuality. These discussions can be particularly sensitive when people of non-marginalised identities are discussing marginalised identities. The anonymous space allowed people from particular identities, and supporters, to defend themselves without being outed. Anonymous online spaces may provide a more equal place for participants without revealing their social identity (Sproull \& Kiesler, 1991), and this helps create a free discussion environment through the autonomous disclosure of personal identity (Zarsky, 2004). Some students saw the anonymity GoSoapBox provided as beneficial:

I found it really interesting to witness an uncensored anonymous conversation between my peers. It opened my eyes up to differing opinions - I wasn’t so much offended by things I 
strongly disagree with, but was reminded by how much viewpoints can vary by. You would never normally get to here[sic] such strong opinions from these people.

One student stated that the anonymity was one of the best things about GoSoapBox. Conversely, anonymity "can curb social inhibitions and result in highly offensive language and views from some writers" (Reader, 2012, pp. 497-498). This, in some cases, led to GoSoapBox being an unsafe space for some students and staff (particularly staff of marginalised identities) and, we believe, also led to trolling behaviour. Hardaker (2010) defines trolling as a computer mediated communication:

[U]ser who constructs the identity of sincerely wishing to be part of the group in question, including professing, or conveying pseudo-sincere intentions, but whose real intention(s) is/are to cause disruption and/or to trigger or exacerbate conflict for the purposes of their own amusement. (p. 237)

For example, in response to the question, "Is Australia still sexist? Give examples”, one student stated: "You have the free will to live in the kitchen and make a sandwich so no it's non-existant [sic]." Another stated: “Are women talking in this discussion ... Yes. So stop complaining.”

Many comments were made in regard to LGBT (lesbian, gay, bisexual, transgender) equality and there were complaints about a lecture which contained content about LGBT people including a mention of transgender children:

Marriage between a man and a woman is traditional and logical. It shouldn't be changed LGBT communities can continue everything as they have always, to me it's unnatural and the law shouldn't be changed.

In regards to [the] complaint, I believe that LGBIT individuals should be treated equally. But I agree that some of the content in last weeks [sic] lecture was too much and unnecessary like saying her young son dresses as female for example.

Race was a topic where students felt free to voice discriminatory opinions:

Half way through the video [indigenous people from the] Northern Territory say they [the Australian Government] are taking funding away not your land! You say that you live off the land then why do you need the funding stay on your land and do the traditional ways.

One student stated: “I’m a strong independent black woman and I don’t need no man.” And was responded to with: "Enjoy your ten year shorter life". On the topic of racism and humour one student stated: "But shouldn't people be able to take a joke even if it is a bit racist? As long as it isn't taken too far?”

Students provided feedback about trolling and anonymity. In response to the question: "What were some of the worst things about GoSoapBox?”, they answered:

- People using the anonymity to be offensive and/or stupid in their comments

- Immature and offensive comments

- Having to read offensive comments by ignorant individuals - you think you're funny you're not

- Trolling

- Anonymity

As mentioned, about $60 \%$ of students who responded to the survey question found GoSoapBox created some degree of unsafe space or offensive environment. One student also made a comment about feeling unsafe:

As a 'larger' girl I am feeling very targeted by the discussions and feedback in both the lectures and the tutorials. I can feel myself sinking into the seat when students are constantly talking about obesity etc. 
In addition to trolling, there was straight-forward silliness, such as:

- When people talk about alcohol I feel like going to the pub. That is all.

- $\quad$ Let's face it, you can't domesticate cats because they are evil :)

- How do I get more swag?

- Do you have a moment to discuss our lord and saviour Poseidon?

- Hi my name is Josh McClure and I'm sitting in the row 6 in the middle with a blue jumper, I am single and ready to mingle.

This reduced as the semester progressed, but it was something that the students remembered and provided feedback about. In response to the question: "What were some of the worst things about GoSoapBox" they stated:

- The go soap box [sic] was really fun and interesting, except for people being silly on it

- $\quad$ People being silly

- Stupidity - just wastes lecture time having to scroll through the rubbish. Detracts from the sound discussion or argument taking place

- People asking irrelevant questions, looking to get a laugh by submitting something offensive and/or stupid

One student even stated that one of the best things about GoSoapBox was "getting to make jokes behind a mask.” These concerns relate to other issues of distraction that the students raised:

[GoSoapBox] Seems to make people talk more during the lecture in response to whatever is being posted - more distracting than the normal low talk rate!

I feel really bad typing away on my phone during a lecture. Also just reading comments during the lecture makes it hard to focus on what the lecturer is saying. Maybe if we were encouraged to use go soapbox[sic] after the lecture?

The effects of distraction on learning are well documented (e.g., Foerde, Knowlton, \& Poldrack 2006; Titsworth, 2001; Watkins, Watkins, Craik, \& Mazuryk, 1973; Wicker \& Holley, 1971), some of this work is even specific to online class discussion and in-class computer use (e.g., Dooley \& Wickersham, 2007; Fried, 2008; Hembrooke \& Gay, 2003).This demonstrates another way GoSoapBox negatively affected learning.

\section{Discussion and conclusion}

This study set out with the key aims of finding out the extent to which a large, multidisciplinary, undergraduate health and sociology cohort would engage with an SRS such as GoSoapBox in a way that exposed and explained their learning processes within this specific tertiary context. The study was guided by SLT, and sought to unearth the extent to which modelling and reciprocity was used during student engagement as they worked to better understand unit concepts and content.

In addressing our first research question regarding the extent to which GoSoapBox enhanced student engagement, we found that while the survey results did not indicate that GoSoapbox was increasing lecture attendance, about a third of students said that the tool greatly influenced their likelihood to engage with the content, and about half said that the discussion boards were the most conducive to their learning. While two thirds reported that it did not always provide a safe-space for speaking and learning, they did concur that it was always interesting to hear the views of their peers. In line with Junco et al. (2001), we found that the use of this technology can contribute to improved frequency and quality of interactions and encourage a collaborative active learning environment. Our findings regarding anonymity support previous research (Banks, 2006; Caldwell, 2007; Draper \& Brown, 2004; Durbin \& Durbin, 2006; Hoppenfeld, 2012; Jones, Connolly, \& Read , 2001; Kay \& LeSage, 2009; Reader, 2012; Siau, Sheng, \& Nah 2006; Sproull \& Kiesler, 1991; Ward 2003; Zarsky 2004). 
Our research found that in relation to our second research question regarding the contribution of an anonymous online qualitative component to learning about potentially socially and culturally sensitive topics, that students appreciated the anonymity of GoSoapBox, as it enabled them to ask questions, respond to questions, and discuss things without risking embarrassment. However, we also found drawbacks of anonymity with a proliferation of trolling contributing to the creation of an unsafe learning environment for marginalised students, which can negatively affect their learning. The application of the SLT framework revealed the interactive and learning processes elicited via use of GoSoapBox as an aid for communication and debate during the traditional lecture format. Specifically, GoSoapBox allows students to observe and gain insights to how other students perceive and interpret the lecture content. They are able to view and engage in a diverse range of responses to the material and ideas being presented, and then to respond to these by contributing to the online discussions in a manner that either reinforces or challenges the comments that other students have posed. Via a process of filtering or rewarding particular behaviours and communication styles, students both shape and model the in-class debates to socially construct a context for learning through discussion.

Our final pedagogical research question on the applicability of using the SLT framework to understand the effectiveness of GoSoapBox, we found that it is crucial for a diverse range of models to be present if students are to gain both the confidence and the ability to participate in discussions that lead to sustained learning and improved critical thinking. The application of the four key conceptual elements of Bandura's SLT revealed that the majority of students initially watched and assessed the interactions and contributions of the cohort leaders in the attentional process phase. They then made more critical evaluations about what was working and what was not within that communicative learning arena in what Bandura called the retentional processes phase. Many then used the results of these assessments to make contributions of their own in the motor reproduction phase. Finally, as part of the motivational process, students were exposed to many models, behaviours, and communication styles during the semester, ultimately modelling the ones that were driving the cohort forward in terms of their enjoyment in class and their learning.

Future use of GoSoapBox in our unit requires a code of conduct outlining appropriate behaviour to ensure safe spaces, minimise distraction, and increase learning. Moderation by staff and students in applying this code of conduct is also required. Having a practice session at the beginning of semester outside of lecture time might a way to give students the space to familarise themselves with the technology and get any silliness out of their systems. Although not analysed here, we'd also like to have a semester long GoSoapBox consultation session where students can ask questions about assessment and other unit requirements or content. In this session, the anonymous environment may make some students more comfortable to ask questions and answers and responses to common queries would be available for quick reference.

\section{References}

Alqurashi, E. (2016). Self-Efficacy in online learning environments: A literature review. Contemporary Issues In Education Research, 9(1), 45-52. https://doi.org/10.19030/cier.v9i1.9549

Bandura, A. (1977). Social learning theory. New York, NY: General Learning Press.

Bandura, A. (2001). Social cognitive theory: An agentic perspective. Annual Review of Psychology, 52(1), 1-26. https://doi.org/10.1146/annurev.psych.52.1.1

Bandura, A. (2012). On the functional properties of perceived self-efficacy revisited, Journal of Management, 38(1), 9-44. https://doi.org/10.1177/0149206311410606

Banks, D. A. (2006). Reflections on the use of ARS with small groups. In D. Banks (Ed.), Audience response systems in higher education: Applications and cases (pp. 373-386). Hershey, PA: IGI Global. https://doi.org/10.4018/978-1-59140-947-2.ch025

Berger, P. L., \& Luckmann T. (1967). The social construction of reality: A treatise in the sociology of knowledge. Garden City, NY: Doubleday.

Beatty, I. D., \& Gerace, W. J. (2009). Technology-enhanced formative assessment: A research-based pedagogy for teaching science with classroom response technology, Journal of Science Education and Technology, 18(2). 146-162. https://doi.org/10.1007/s10956-008-9140-4

Caldwell, J. E. (2007). Clickers in the large classroom: Current research and best-practice tips. Life Sciences Education, 6(1), 9-20. https://doi.org/10.1187/cbe.06-12-0205 
Chiu, S. (2014). The relationship between life stress and smartphone addiction on taiwanese university student: A mediation model of learning self-efficacy and social self-efficacy. Computers in Human Behavior, 34(May 2014), 49-57. https://doi.org/10.1016/j.chb.2014.01.024

Corriveau, K. H. (2016). Preschoolers' preference for syntactic complexity varies by socioeconomic status. Child Development, 87(5), 1529-1537. https://doi.org/10.1111/cdev.12553

d'Inverno, R., Davis, H., \& White, S. (2003). Using a personal response system for promoting student interaction. Teaching Mathematics and its Applications, 22(4), 163-169. https://doi.org/10.1093/teamat/22.4.163

Dooley, K. E., \& Wickersham, L. E. (2007). Distraction, domination and disconnection in whole-class online discussions. The Quarterly Review of Distance Education, 8(1), 1-8.

Draper, D., \& Brown, M. (2004). Increasing interactivity in lectures using an electronic voting system. Journal of Computer Assisted Learning, 20(2), 81-94. https://doi.org/10.1111/j.13652729.2004.00074.x

Durbin, S. M., \& Durbin, K. A. (2006). Anonymous polling in a engineering tutorial environment: A case study. In D. Banks (Ed.), Audience response systems in higher education: Applications and cases (pp. 373-386). Hershey, PA: IGI Global. https://doi.org/10.4018/978-1-59140-947-2.ch008

Foerde, K., Knowlton, B. J., \& Poldrack R. A. (2006). Modulation of competing memory systems by distraction. Proceedings of the National Academy of Sciences of the United States of America, 103(31), 11778-11783. https://doi.org/10.1073/pnas.0602659103

Fried, C. B. (2008). In-class laptop use and its effects on student learning. Computers \& Education, 50(3), 906-914. https://doi.org/10.1016/j.compedu.2006.09.006

Glassman, M., \& Kang, M. J. (2011). The logic of wikis: The possibilities of the Web 2.0 classroom. Computer-Supported Collaborative Learning, 6(1), 93-112. https://doi.org/10.1007/s11412-011-9107-y

Hardaker, C. (2010). Trolling in asynchronous computer-mediated communication: From user discussions to academic definitions. Journal of Politeness Research: Language, Behaviour, Culture, 6(2), 161285. https://doi.org/10.1515/JPLR.2010.011

Hembrooke, H., \& Gay, G. (2003). The laptop and the lecture: The effects of multitasking in learning environments. Journal of Computing In Higher Education, 15(1), 46-64. https://doi.org/10.1007/BF02940852

Hoppenfeld, J. (2012). Keeping students engaged with web-based polling in the library instruction Session. Library Hi Tech, 30(2), 12-26. https://doi.org/10.1108/07378831211239933

Hauffman, A., Alfonsson, S., Mattsson, S., Forslund, M., Bill-Axelson, A., Nygren, P., \& Johansson, B. (2017). The development of a nurse-led internet-based learning and self-care program for cancer patients with symptoms of anxiety and depression - A part of U-CARE. Cancer Nursing, 40(5), E9E16. https://doi.org/10.1097/NCC.0000000000000402

Jones, C., Connolly, M., \& Read, M. (2001). Group integrative learning with group process support technology. British Journal Of Educational Technology, 32(5), 571-581. https://doi.org/10.1111/1467-8535.00226

Junco, R., Heibergert, G., \& Loken, E. (2011). The effect of Twitter on college student engagement and grades. Journal of Computer Assisted Learning, 27(2), 119-132. https://doi.org/10.1111/j.13652729.2010.00387.X

Kay, R. H., \& Lesage A., (2009). Examining the benefits and challenges of using audience response systems: A review of the literature. Computers \& Education, 53(3), 819-827.

Lantz, M. E. (2010). The use of 'clickers' in the classroom: Teaching innovation or merely an amusing novelty? Computers in Human Behavior, 26(4), 556-561. https://doi.org/10.1016/j.chb.2010.02.014

Matthew, A. F., \& Evans R. (2012, June). Managing distraction in 21st century learning environments. Paper presented at The 15th International First Year in Higher Education Conference, Brisbane. Retrieved from https://eprints.qut.edu.au/53489/2/53489.pdf

Méndez-Coca, D., \& Slisko, J. (2013). Software Socrative and smartphones as tools for implementation of basic processes of active physics learning in classroom: An initial feasibility study with prospective teachers. European Journal of Physics Education, 4(2), 17-24. Retrieved from http://dergipark.gov.tr/download/article-file/62733

Reader, B. (2012). Free press vs. free speech? The rhetoric of “civility” in regard to anonymous online comments. Journalism \& Mass Communication Quarterly, 89(3), 495-513. https://doi.org/10.1177/1077699012447923

Schunk, D. (2012). Learning theories: An educational perspective (6th ed.). Boston, MA: Pearson Education. 
Siau, K., Sheng, H., \& Nah, F. F. (2006). Use of classroom response system to enhance classroom interactivity. IEEE Transactions on Education, 49(3), 398-403. Retrieved from https://doi.org/10.1109/TE.2006.879802

Sprague, D. E. W., \& Dahl D. W. (2009). Learning to click: An evaluation of the personal response system clicker technology in introductory marketing courses. Journal of Marketing Education, 32(1), 93-103. https://doi.org/10.1177/0273475309344806

Sproull, L., \& Kiesler, S. (1991). Connections: New ways of working in the networked organization. Cambridge, MA: MIT Press.

Snyder, S, \& Fisk, T. (2016). Applying Bandura's model to identifying sources of self-efficacy of teaching artists. Research in The Schools, 23(2), 38-50.

Titsworth, B. S. (2001). The effects of teacher immediacy, use of organizational lecture cues, and students' notetaking on cognitive learning. Communication Education, 50(4), 283-297. https://doi.org/10.1080/03634520109379256

Ward, D. (2003). The classroom performance system: The overwhelming research results supporting this teaching tool and methodology. [White Paper]

Watkins, M. J., Watkins, O. C., Craik, I. F., \& Mazuryk, G. (1973) Effect of nonverbal distraction on short-term storage. Journal of Experimental Psychology, 101(2), 296-300. https://doi.org/10.1037/h0035252

Wicker, F. W., \& Holley, F. M. (1971) Distraction modality and stimulus modality in paired-associate learning. Psychonomic Science, 25(4), 218-220. https://doi.org/10.3758/BF03329098

Zarsky, T. (2004). Thinking outside the box: Considering transparency, anonymity, and pseudonymity as overall solutions to the problems of privacy in the internet society. University of Miami Law Review, 58(4), 991-1044. Retrieved from https://repository.law.miami.edu/umlr/vol58/iss4/13

Corresponding author: Julie-Anne Carroll, jm.carroll@qut.edu.au

Australasian Journal of Educational Technology @ 2018.

Please cite as: Carroll, J-A., Sankupellay, M., Newcomb M., Rodgers, J. \& Cook, R.. (2018). Gosoapbox in public health tertiary education: a student response system for improving learning experiences and outcomes. Australasian Journal of Educational Technology, 34(5), 58-71.

https://doi.org/10.14742/ajet.3743 